\title{
A Novel Chaotic Modulation Scheme in a Cooperative Relaying System
}

\author{
K.Thilagam \\ Research Scholar \\ Department of ECE \\ Pondicherry Engg. College
}

\author{
K.Jayanthi \\ Associate Professor \\ Department of ECE \\ Pondicherry Engg. College
}

\begin{abstract}
In recent years, the demand for new-generation wireless networks has spurred research on cooperative communication, a powerful physical layer technique to combat fading in wireless relaying scenario. Recently, chaos based communication has been proved to exhibit excellent features which suits better for the emerging wireless networks. Different chaos based modulation schemes has been analyzed with the recent literatures, of which the code shifted differential chaos shift keying (CS-DCSK) and Quadrature chaos shift keying (QCSK) schemes are proved to be the better schemes. By combining the advantage of aforementioned CS-DCSK and QCSK scheme, a novel modulation scheme called code shifted Quadrature chaos shift keying (CS-QCSK) is reported in our earlier work [21] and its suitability in a cooperative relaying scenario with a single relay node is tested in this paper. The novel CS-QCSK scheme is implemented in the nodes of the cooperative relaying system to upgrade the system performance. The simulation results have validated the effectiveness of the proposed scheme by offering better BER performance, minimum outage probability and increased spectral efficiency compared to the non-cooperative transmission method.
\end{abstract}

\section{General Terms}

Cooperative communication, chaos based modulation schemes, physical layer techniques.

\section{Keywords}

Cooperative communication, Chaotic modulation, Code shifted Quadrature chaos shift keying (CS-QCSK), Decode and Forward (DF) protocol, Bit error rate (BER), outage probability, spectral efficiency.

\section{INTRODUCTION}

Cooperative communication is an interesting research topic in wireless communication for it achieves distributed spatial diversity, wider coverage, low transmit power and reduced interference. Recently, a good number of researches are also focused on chaotic carrier modulation. A promising technique which exploits the advantages of aforementioned schemes by combining chaos based modulation in cooperative relaying attracts a widespread attention in the literature. In [1], the authors discuss the application of adaptive modulation concept to enhance the performance of cooperative techniques and to improve the spectral efficiency of the amplify and forward system. The signal designs such as space time codings / modulations to achieve full cooperative diversity and an overview of the efforts on combating the time and frequency asynchronism of the cooperative communication network has been discussed in [2]. The measured bit error rate performance of a three node cooperative communication system operating with a maximum ratio combining (MRC) technique and two cooperative coded schemes using hard and soft decision decoding has been discussed in [3]. In [4], the authors present a Double-differential (DD) modulation for amplify and forward (AAF) cooperative communications system that works well with carrier offsets and further the numerical power allocation improves the performance of the system. The decode-remodulate-and-forward (D-ReM-F) cooperative modulation scheme with higher signal constellations and the capacity analysis for different protocol under different fading condition has been discussed in [5]. In [6], the authors' details about the cooperative relaying schemes like symmetric and asymmetric downlink communications which employs hierarchical modulation schemes. The modulation diversity scheme for uncoded cooperative communication in wireless networks with DF protocol and the power allocation factor for performance improvement in the system have been portrayed in [7]. In [8], the modulation scheme supported with the knowledge of SNRs of all links in a simple DF cooperative communication system and proposed with the two rules, namely the exact rule and the approximate rules has been presented. The performance of adaptive modulation schemes applied both by the source and the relay in cooperative communications with perfect feedback and the impact of noisy feedback channels has been analyzed in [9]. A novel combiner capable of collecting full diversity with DF for any coherent modulation termed cooperative MRC (CMRC) which offers a high performance demodulation with less-complexity has been discussed in [10].

A system combining DCSK / FM- DCSK with cooperative communication strategy effectively enhancing the system performance over multipath fading channels has been discussed in [11]. Quadrature CSK (QCSK) is a multilevel version of DCSK, based on the generation of an orthogonal of chaotic functions. It allows an increase in data rate by occupying same bandwidth with respect to DCSK has been discussed in [12]. The study of multipath performance of the frequency-modulated DCSK (FM-DCSK) system has been analyzed in [13]. In [14],[15] the performance of the DCSK system over a channel with Rayleigh fading or Ricean fading has been discussed with the necessity to model the effects of multipath delay spread as well as fading. A multiple-access technique with differential chaos shift keying using a one dimensional iterative map to generate the chaotic signals for all users and similar average data rates for the users has been 
discussed in [16]. In [17], the author explained the FM-QCSK generation of inherent wideband signal with constant energy per bit, which can be achieved by using the combination of frequency modulation with QCSK. The high-level constellation in FM-QACSK which can improve the speed of chaos shift keying has been examined in [18]. The DCSK system incorporated with two-user cooperative diversity technique and multiple accessing has been adopted and discussed in [19]. CS-DCSK uses code domain approach, the reference and the information bearing signals are transmitted at the same time slots, it offers better BER performance and bandwidth efficiency, as discussed in [20]. The CS-QCSK modulation scheme which is a combination of CS-DCSK and QCSK modulation schemes with the higher data rates and offering the advantage of both the CS-DCSK and QCSK schemes has been discussed in [21]. In this paper, the CS-QCSK modulation scheme discussed in [21] is to check for the suitability of CS-QCSK in a cooperative relaying system.

The remaining part of the paper is organized as follows: In section 2, the system model of the proposed cooperative relaying scheme is elaborated. In section 3, the code shifted quadrature chaos shift keying scheme is discussed analytically with the bit error rate expressions. Simulation results are discussed in Section 4. Section 5, deals with the conclusion of the paper.

\section{SYSTEM MODEL DESCRIPTION}

Consider a cooperative communication network consisting three nodes namely: the source(S) node, $\operatorname{relay}(\mathrm{R})$ node and destination (D) node, with each having a single antenna as referred in fig. 1 . Let ' $h_{s, r}$ ' be the fading channel between the $\mathrm{S}$ and $\mathrm{R}, \mathrm{h}_{\mathrm{s}, \mathrm{d}}$ ' be the fading channel between $\mathrm{S}$ and $\mathrm{D}$, and ' $h_{r, d}$ ' be the fading channel between $R$ and $D$. Cooperative protocol strategies are classified by the operation at $\mathrm{R}$ and here it is assumed to be DF protocol, where $\mathrm{R}$ decodes the received signal, re-encodes, and transmits to $\mathrm{D}$.

Assumptions carried out in the work are:

(i) Rayleigh fading for all the channels

(ii) The perfect channel state information is assumed to be known at the receiver.
(iii)
Half duplex communication

(iv) Cellular mobile environment.

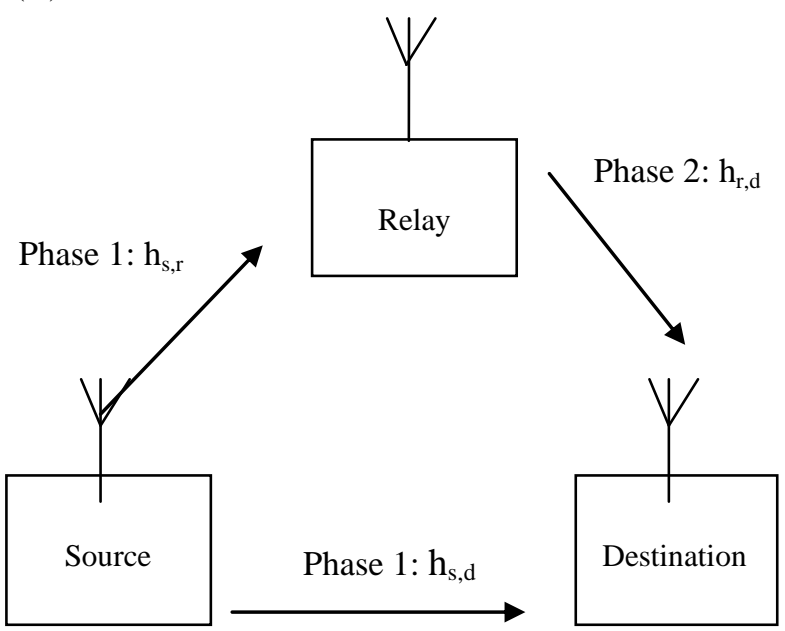

Figure 1: Cooperative Communication for single relay with the Implementation of code shifted quadrature chaos shift keying scheme in the nodes
Internal structures of the source node, relay node and destination node which are shown in Figure 2, Figure 3 and Figure 4.Referring to the Figure 2, the source node consists of a block called CS-QCSK modulator block. The operation at the source node is, the input bit streams are given as input to the CS-QCSK modulator and in the modulator the bits are mapped according to the constellation and generates the CS-QCSK modulated signal. Then, the modulated signal is launched over the transmitting antenna.

Input Bit

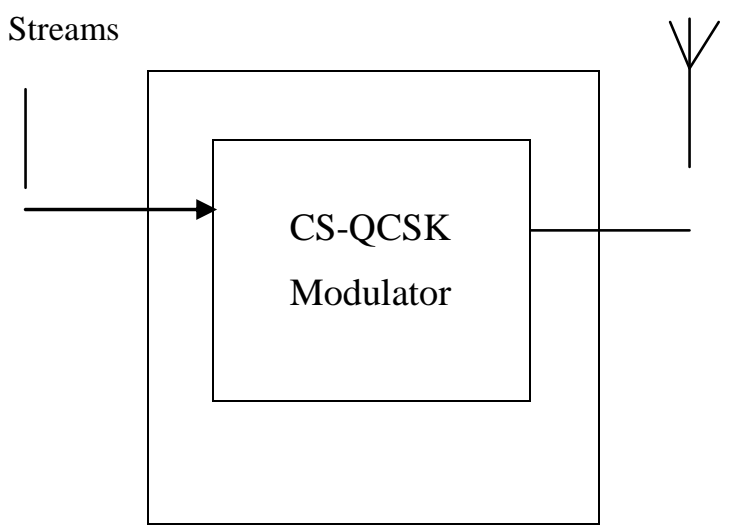

Figure 2: Source node

Overheard

Signals

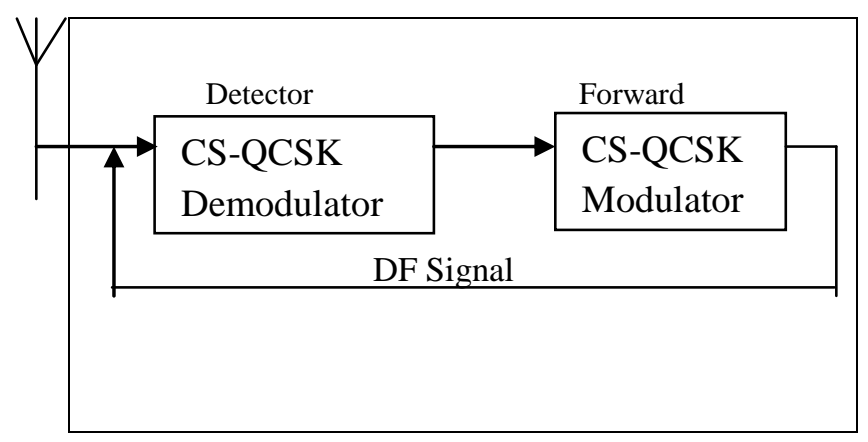

Figure 3: Relay node

Referring to the Figure 3, the relay node consists of two blocks (i.e.) the detector block and forward block. The detector block employs a CS-QCSK demodulator and the forward block performs CS-QCSK modulation. The process involved is, the relay receives the overheard signals and it is given to the detector block. In detector block, the signal is demodulated. Then the detector output is given as input to the forward block. The forward block re-modulates the input signal according to CS-QCSK constellation and generates the Detect and Forward (DF) signals. Further, the DF signal is launched over the transmitting antenna. 


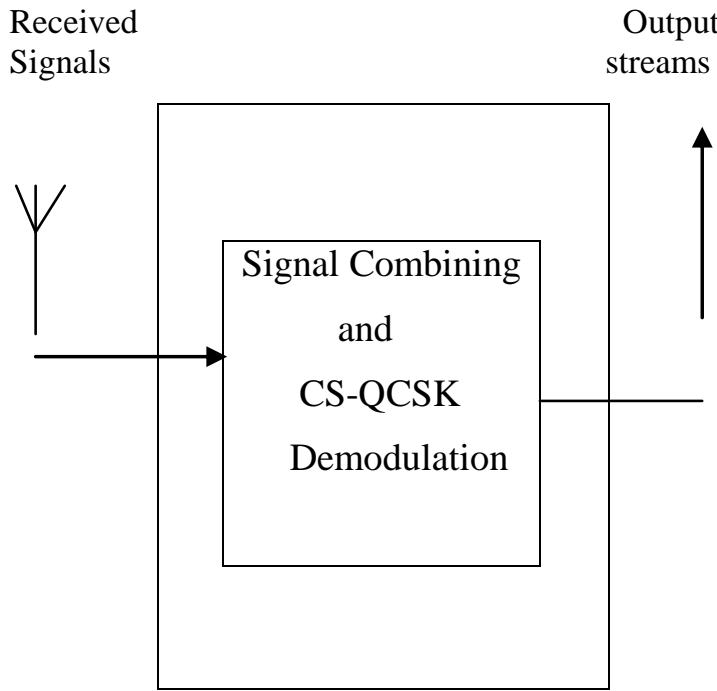

Figure 4: Destination node

Referring to the Figure 4, the destination node consists of a block called signal combining with CS-QCSK demodulation block. The process involved is, the signals are received and then combined using maximum ratio combiner and detected using CS-QCSK demodulator. Finally the output bit streams are obtained.

The cooperation strategy is modelled with two orthogonal phases to avoid the interferences. In phase 1 , source sends information to the destination and the information is received by the relay at the same time. In phase 2 , the relay helps the source by forwarding or retransmitting the information to the destination. In phase 1, the source broadcasts its information to both the destination and the relay. The received signals at the destination and the relay are obtained as,

$$
\begin{aligned}
& \boldsymbol{y}_{s, d}=\sqrt{\boldsymbol{P}} \boldsymbol{h}_{s, d} \boldsymbol{x}+\boldsymbol{n}_{s, d} \\
& \boldsymbol{y}_{s, r}=\sqrt{\boldsymbol{P}} \boldsymbol{h}_{s, r} \boldsymbol{x}+\boldsymbol{n}_{s, r}
\end{aligned}
$$

Where, ' $\mathrm{P}$ ' is the total transmit power at the source, ' $\mathrm{x}$ ' is the transmitted information symbol, ' $n_{\mathrm{s}, \mathrm{d}}$ ', ' $\mathrm{n}_{\mathrm{s}, \mathrm{r}}$ ' and ' $\mathrm{n}_{\mathrm{r}, \mathrm{d}}$ ' are the additive noises, ' $h_{\mathrm{s}, \mathrm{d}}$ ', ' $\mathrm{h}_{\mathrm{s}, \mathrm{r}}$ ' and ' $\mathrm{h}_{\mathrm{r}, \mathrm{d}}$ ' are the channel coefficient from source to destination, source to relay and relay to destination respectively. They are modeled as zero-mean, complex Gaussian random variables with variances $\sigma_{s, d}^{2}, \sigma_{s, r}^{2}$ and $\sigma_{r, d}^{2}$ respectively. With the variance ' $\mathrm{N}_{\mathrm{o}}$ ' the noise terms ' $\mathrm{n}_{\mathrm{s}, \mathrm{d}}$ ', ' $\mathrm{n}_{\mathrm{s}, \mathrm{r}}$ ' and ' $\mathrm{n}_{\mathrm{r}, \mathrm{d}}$ ' are modelled as zero-mean, complex Gaussian random variables. In phase 2 , the relay forwards a processed version of the source signal to the destination and this is obtained as,

$$
\boldsymbol{y}_{r, d}=\boldsymbol{h}_{r, d} q\left(\boldsymbol{y}_{s, r}\right)+\boldsymbol{n}_{r, d}
$$

the function $\mathrm{q}$ (.) depends on which processing is implemented at the relay node. The mutual information in terms of channel fades for decode and forward transmission is given by,

$I_{D F}=\frac{1}{2} \min \left\{\log \left(1+\Gamma\left|\boldsymbol{h}_{s, r}\right|^{2}\right), \log \left(1+\Gamma\left|\boldsymbol{h}_{s, d}\right|^{2}+\Gamma\left|\boldsymbol{h}_{r, d}\right|^{2}\right)\right\}$

Considering rayleigh fading channel, the outage probability can be written as,
$\operatorname{Pr}\left[I_{D F}<R\right]=\operatorname{Pr}\left\{\left|h_{s, r}\right|^{2}<\frac{2^{2 R}-1}{\Gamma}\right\}+\operatorname{Pr}\left\{\left|h_{s, r}\right|^{2}>\frac{2^{2 R}-1}{\Gamma}\right\} \operatorname{Pr}\left\{\left|h_{s, d}\right|^{2}+\left|h_{r, d}\right|^{2}<\frac{2^{2 R}-1}{\Gamma}\right\}$

Averaging over the channel conditions, the outage probability

for decode and forward is given by,

$\operatorname{Pr}\left[I_{D F}<R\right] \square \frac{1}{\sigma_{s, r}^{2}} \frac{2^{2 R}-1}{\Gamma}$

Where, $\Gamma=\mathrm{P} / \mathrm{N}_{0}$ and ' $\mathrm{R}$ ' is the transmission rate. The next section deals with the analytical description of CS-QCSK modulation scheme in detail.

\section{CHAOTIC MODULATION SCHEME}

The aim of this section is to illustrate the practical importance of CS-QCSK modulation scheme which is highlighted with the modulated expression and bit error rate expressions for AWGN channel and Rayleigh channel conditions.

\subsection{CS-QCSK Scheme}

In CS-QCSK, both reference signal and information signal (symbol ' $\mathrm{S}$ ') is transmitted in same time slot. However they are separated using walsh codes. The CS-QCSK transmitted signal is given by,

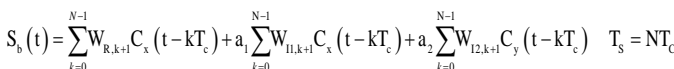

Where $a_{1} \in\{-1,1\}, a_{2} \in\{-1,1\}$ is mapped from $b \in\{0,1\}$ which is the information bit to be transmitted. This scheme uses different Walsh code for the reference and information signal, where $\mathrm{W}_{\mathrm{R}, \mathrm{k}+1}$ represent the Walsh code for reference signal and $\mathrm{W}_{\mathrm{II}, \mathrm{k}+1}, \mathrm{~W}_{\mathrm{I} 2 \mathrm{k}+1}$ represent the Walsh code for information signal, $C(t)$ is the chaotic signal with duration of $\mathrm{T}_{\mathrm{c}}$. Both the reference and the information signal are transmitted in the same time slot as given in equation (7). The orthogonality of the signal is assured by walsh code sequences, therefore the reference and the information signal are independent of the chaotic carrier. The description about chaotic modulator / demodulator discussed under this section can be referred in the earlier paper [21].The error rates associated with CS-QCSK under AWGN and Rayleigh environment is given below,

The Bit error rates under AWGN channel is given as,

$$
B E R_{C S-Q C S K}=\frac{1}{2} \operatorname{erfc}\left(\frac{3\left(E_{b} / N_{0}\right)}{\sqrt{\frac{3}{4} K+\frac{1}{2} K+2\left(E_{b} / N_{0}\right)}}\right)
$$

The Bit error rates under Rayleigh fading is given as,

$$
B E R_{C S-Q C S K}=\frac{1}{2} \operatorname{erfc}\left(\frac{3\left(\gamma_{b}\right)}{\sqrt{\frac{3}{4} K+\frac{1}{2} K+2\left(\gamma_{b}\right)}}\right)
$$

Where ' $\mathrm{E}_{\mathrm{b}}$ ' is the bit energy, ' $\mathrm{N}_{\mathrm{o}}$ ' is the noise power spectral density, ' $\mathrm{K}$ ' is the spread factor, ' $\gamma_{\mathrm{b}}$ ' is the product of $\left(\mathrm{E}_{\mathrm{b}} / \mathrm{N}_{\mathrm{o}}\right)$ and the gain of the propagation path. The next section deals with the simulation analysis of the proposed schemes with various validating factors.

\section{SIMULATION ANALYSIS}

The simulation analysis for the proposed method is performed with the following parameters and it is tested in MATLAB 7.10. This section discusses the simulation analysis of the 
proposed scheme using the parameters mentioned in Table 1. The complexity analysis of the CS-QCSK scheme is tested initially and further the performance analysis of the proposed scheme in a cooperative relaying scenario is analyzed in terms of parameters like outage probability, spectral efficiency, BER etc. The superiority of the CS-QCSK chaotic scheme has been already tested. The readers are requested to refer to [21].

Table 1. Simulation Parameters

\begin{tabular}{|c|c|}
\hline PARAMETERS & VALUES / TYPES \\
\hline Number of transmitted bits & $2 \times 10^{4}$ \\
\hline Type of antenna system & SISO \\
\hline Modulation scheme & CS-QCSK \\
\hline Number of Transmitter & 1 \\
\hline Number of receiver & 1 \\
\hline Number of relay & 1 \\
\hline Power allocation & Ps=Pr= (1/2)P $=0.5$ \\
\hline Relay location & Dsr=Dsd=(1/2)Dsd=0.5 \\
\hline Path loss exponent & 2 \\
\hline Doppler frequency & 0.01 \\
\hline Channel Type & Rayleigh fading \\
\hline
\end{tabular}

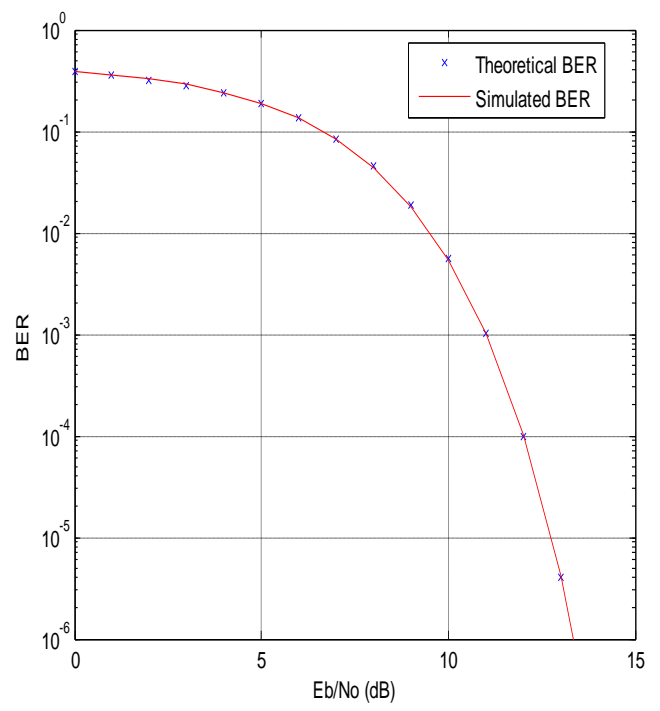

Figure 5: BER performance of CS-QCSK scheme for Rayleigh multipath channel.

The fig.5 reveals the BER performance of CS-QCSK scheme obtained for both theoretical and simulated values.The BER values calculated from the analytical expression and the simulation are very similar and both the graph converge with each other.For the BER value of $10^{-3}$, approximately the required $(\mathrm{Eb} / \mathrm{No})$ value is $11 \mathrm{~dB}$.

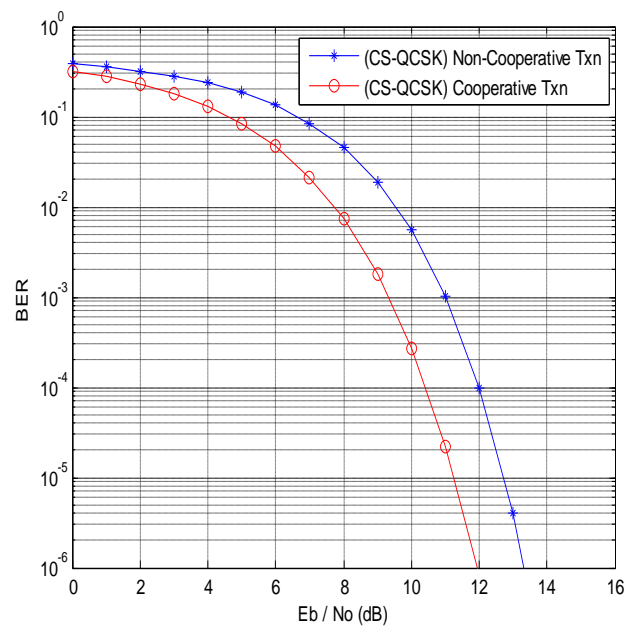

Figure 6: BER performance analysis of the CS-QCSK scheme in Cooperative relaying scenario

It is inferred that, for a fixed BER of $10^{-3}$, the cooperative transmission method requires approximately $9 \mathrm{~dB}$ and non-cooperative transmission scheme requires $11 \mathrm{~dB}$. This is due to the fact that a cooperative mode has enabled the link to be good even under adverse situations. The BER performance of the proposed CS-QCSK scheme in cooperative transmission has better performance compared to the CS-QCSK scheme in non-cooperative transmission.

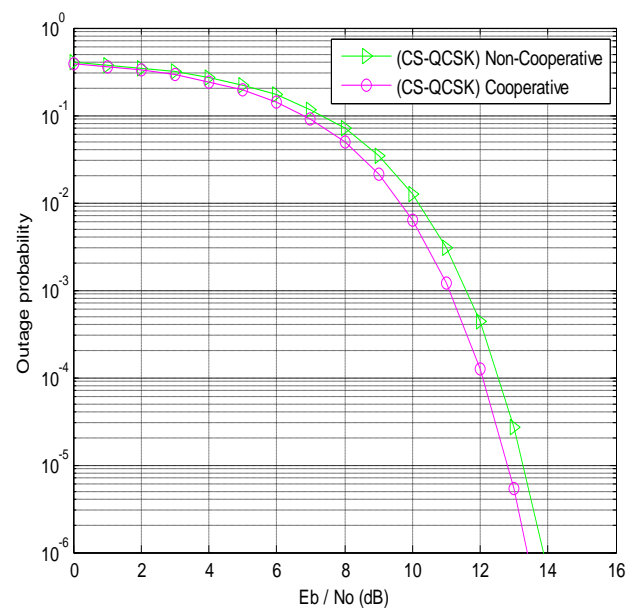

Figure 7: Outage probability vs. Eb / no of CS-QCSK in cooperative relaying scenario

The outage probability analysis for the CS-QCSK scheme in cooperative relaying transmission and non-cooperative transmission is plotted. From fig. 7, it is inferred that, for a fixed $\mathrm{Eb} / \mathrm{No}$ of $12 \mathrm{~dB}$ the CS-QCSK scheme in cooperative transmission method and non-cooperative transmission method has the outage probability of approximately $1 \times 10^{-4}$ and $1.6 \times 10^{-4}$ respectively. This proves the effectiveness of the cooperative mode of transmission. 


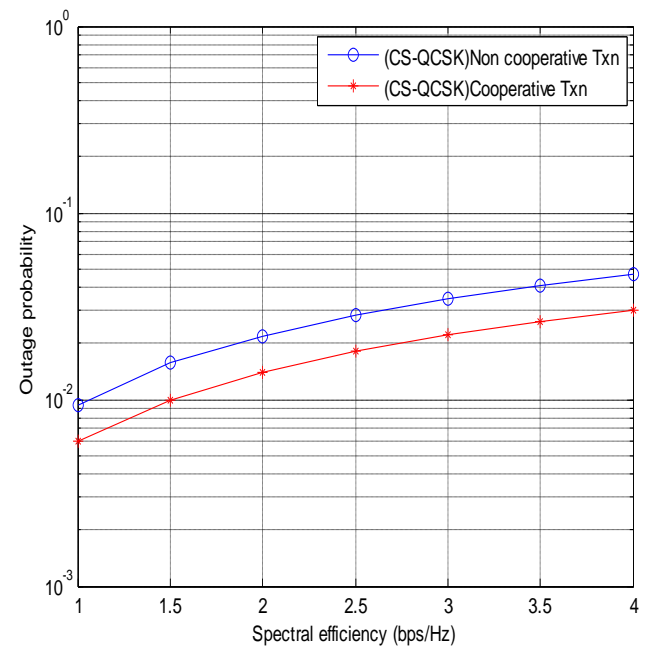

Figure 8: Outage Probability versus Spectral Efficiency of CSQCSK scheme in Cooperative relaying scenario

The next attempt was to check out for another critical 'QoS' parameter namely outage probability and its relation with spectral efficiency. From fig. 8, it can be concluded that for a fixed spectral efficiency of $2 \mathrm{bps} / \mathrm{Hz}$ the proposed CS-QCSK scheme in cooperative transmission method has the outage probability of $1.384 \times 10^{-4}$ and in non-cooperative transmission method has the outage probability of 0.0105 . Then, for fixed outage probability of $10^{-2}$, the spectral efficiency for cooperative transmission method and non-cooperative transmission is $1.5 \mathrm{bps} / \mathrm{Hz}$ and $1.1 \mathrm{bps} / \mathrm{Hz}$ respectively. The CS-QCSK scheme in cooperative transmission has minimum outage probability and increased spectral efficiency compared to a Non-cooperative transmission method. According to the system capacity condition, as the spectral efficiency increases the outage probability also gets increased. It is obvious from the figure 5 that tradeoffs always exist between outage probability and spectral efficiency.

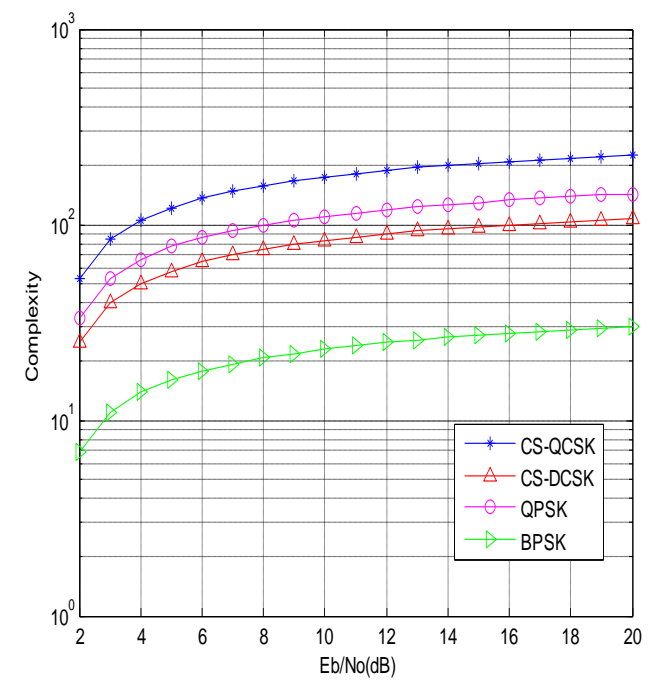

Figure 9: Complexity analysis for the proposed CS-QCSK scheme and Conventional modulation scheme

Finally, the complexity analysis graphs were plotted for the proposed CS-QCSK scheme and conventional modulation scheme. From fig. 9, it is inferred from the graph that, for a fixed $E_{b} / N_{o}$ of $4 \mathrm{~dB}$ the number of operations performed by the proposed CS-QCSK scheme is 100 (approx) and for the conventional modulation schemes CS-DCSK, QPSK and BPSK the number of operations required are approximately 50,70 and 15 respectively. The complexity analysis expression is derived for the proposed scheme and the analysis is done through it. Though the proposed scheme has more computational complexity compared to the conventional schemes, it offers other benefits like improved BER performance, spectral efficiency and higher data rate.

\section{CONCLUSION}

In this paper, an improved chaos-based modulation, CS-QCSK technique is recommended for a cooperative relaying scenario. A single relay cooperative communication with DF protocol is considered. The CS-QCSK scheme, avoids the use of delay lines in the receiver units. It transmits the reference and information signals in the same time slot and offers an increase in data rate, better BER performance and bandwidth efficiency. However, the proposed logic suffers from a marginal increased system complexity which is analyzed through simulation and compared with the conventional modulation schemes. Simulation analysis is carried out and the results shows that the proposed scheme achieves better BER performance, minimum outage probability and increased spectral efficiency, which is the need of the hour for the future wireless communication systems. The same work can also be extended to a multi-relay node scenario, which would certainly yield a satisfactory performance with an exception in the complexity involved.

\section{REFERENCES}

[1] Wang and Xia, "Asynchronous cooperative communication systems: A survey on signal designs", Science China Information Sciences, August 2011.

[2] Ehsan Yazdian,Mohammad Reza Pakravan, Adaptive Modulation Technique for Amplify and Forward Cooperative Diversity and Fairness Analysis, IEEE International Conference on Telecommunications, ICT June 2008.

[3] Michael Knox, Elza Erkip, Kshitij Kumar Singh, "Cooperative coding implementation at the physical layer", IEEE International Conference Acoustics Speech and Signal Processing (ICASSP), March 2010.

[4] Manav R.Bhatnagar,and Are Hjorunges, “Amplify based double differential modulation for Cooperative communications", Proceedings in IEEE International conference on COMmunication Systems And NETworks, COMSNETS'09

[5] Jun Yang, Philips, Briarcliff Manor, Ghosh, M, "A Cooperative Modulation Scheme for Wireless Relay Networks", IEEE Vehicular Technology Conference proceedings,pp. 1628 - 1632, April 2007.

[6] Roderick Jaehoon Whang, Saransh Malik, Intae Hwang, Huaping Liu, "Cooperative Multiuser Relay Communications Employing Hierarchical Modulation", Wireless Personal Communications, Springer Proceedings, April 2012.

[7] Kiyani, N.F., Eindhoven, Rizvi, U.H. , Dolmans, G., "Modulation diversity benefits in cooperative communications", IEEE Wireless Telecommunications Symposium (WTS), pp. 1-5, April 2010. 
[8] Chih- Yung Song, Min-Kuan Chang and Guu-Chang Yang,"Adaptive Modulation in Decode-and-Forward (DF) Cooperative Communications , IEEE Vehicular Technology Conference (VTC Spring) Proceedings, pp. 1-5,May 2012.

[9] Chuyi Qian, Yi Ma and Tafazolli, "Adaptive modulation for cooperative communications with noisy feedback, IEEE International Wireless Communications and Mobile Computing Conference (IWCMC), pp 173 - 177, July 2011.

[10] Tairan Wang, Alfonso Cano, Georgios B. Giannakis, and J. Nicholas Laneman, High-Performance Cooperative Demodulation With Decode-and-Forward Relays, IEEE Transactions On Communications, Vol. 55, No. 7, JULY 2007.

[11] Jing Xu, Weikai Xu, Lin Wang, Guanrong Chen, "Design and Simulation of a Cooperative Communication System Based on DCSK/FM-DCSK", IEEE International Symposium on Circuits and Systems (ISCAS) Proceedings, pp.2454 - 2457, June 2010.

[12] Z. Galias and G. M. Maggio, "Quadrature chaos-shift keying: Theory and performance analysis," IEEE Trans.Circuits Syst. I, Fundam.Theory Appl., vol. 48, no. 12, pp. 1510-1519, Dec. 2001.

[13] G. Kolumbán and G. Kis, "Multipath performance of FM-DCSK chaotic communications system," in Proc. IEEE Int. Symp. Circuits and Systems, Geneva, Switzerland, pp. 433-436, May 2000.

[14] S. Mandal and S. Banerjee, "Performance of differential chaos shift keying over multipath fading channels," in
Proc. Indian Nat. Conf. Nonlinear Systems and Dynamics, Kharagpur, India, Dec. 2003.

[15] Xia, Y.,Tse,C.K.\& Lau, F.C.M, "Performance of differential chaos shift keying digital communication systems over a multipath fading channel with delay spread,'IEEE Trans. Circuits Syst.-II 51,680-684,2004.

[16] F. C. M. Lau, M. M. Yip, C. K. Tse, and S. F. Hau, "A Multiple-Access Technique for Differential Chaos-Shift Keying" IEEE Trans.Circuits Syst. I, Fundam.Theory Appl., vol. 49, no. 1, January 2002.

[17] YiWei Zhang, Xubang Shen and Yi Ding, "Design and performance analysis of an FM- QCSK chaotic communication system," International Conference on Wireless Communications, Networking and Mobile Computing, China, pp: 1-4, Sept. 2006.

[18] Jiamin Pan, He Zhang, "Design of FM-QACSK Chaotic Communication System" IEEE trans. Wireless Communications \& Signal Processing, 2009.

[19] Wang,L.,Zhang,C.\& Chen, G. "Performance of an SIMO FM-DCSK communication system," IEEE Trans.Circuits Syst.-II, 55, 457-461,2008.

[20] G.Koluban,W.K.Xu and L.Wang, "A Novel differential chaos shift keying modulation scheme," International journal of Bifurcation and chaos, Vol.21,No.3,pp799814,2011

[21] Thilagam.K and Jayanthi.K, "Analytical Evaluation of a Hybrid Chaotic Shift Keying [CS-QCSK] inCDMA System", International Journal of Computer Applications (IJCA), Volume 54- No.15, September 2012 